\title{
MAHLER MEASURES OF POLYNOMIALS THAT ARE SUMS OF A BOUNDED NUMBER OF MONOMIALS
}

\author{
EDWARD DOBROWOLSKI AND CHRIS SMYTH
}

\begin{abstract}
We study Laurent polynomials in any number of variables that are sums of at most $k$ monomials. We first show that the Mahler measure of such a polynomial is at least $h / 2^{k-2}$, where $h$ is the height of the polynomial. Next, restricting to such polynomials having integer coefficients, we show that the set of logarithmic Mahler measures of the elements of this restricted set is a closed subset of the nonnegative real line, with 0 being an isolated point of the set. In the final section, we discuss the extent to which such an integer polynomial of Mahler measure 1 is determined by its $k$ coefficients.
\end{abstract}

\section{Statement of RESUlts}

For a polynomial $f(z) \in \mathbb{C}[z]$, we denote by $m(f)$ its logarithmic Mahler measure

$$
m(f)=\int_{0}^{1} \log \left|f\left(e^{2 \pi i t}\right)\right| d t,
$$

and write $M(f)=\exp (m(f))$ for the (classical) Mahler measure of $f$. Although first defined by D.H. Lehmer [7, its systematic study was initiated by Kurt Mahler [8, 9, 10].

Let $h(f)$ denote the height of $f$ (the maximum modulus of its coefficients). Our first result relates these two quantities.

Theorem 1. For an integer $k \geq 2$, let

$$
f(x)=a_{1} z^{n_{1}}+\cdots+a_{k-1} z^{n_{k-1}}+a_{k} \in \mathbb{C}[z] \text { with } n_{1}>n_{2}>\cdots>n_{k-1}>0
$$

be a nonzero polynomial. Then

$$
M(f) \geq \frac{h(f)}{2^{k-2}} .
$$

The example $(z+1)^{k-1}$ shows that the constant $1 / 2^{k-2}$ in this inequality cannot be improved to any number bigger than $1 /\left(\begin{array}{c}k-1 \\ \lfloor k-2) / 2\rfloor\end{array}\right)$ (which $\sim \sqrt{2 \pi k} / 2^{k}$ as $k \rightarrow \infty$ ).

The inequality for the special case $\left(n_{1}, n_{2} \ldots, n_{k-1}\right)=(k-1, k-2, \ldots, 1)$ (i.e., a polynomial of degree $k-1$ ) follows from a result of Mahler [10, equation (6)].

In the other direction we have from (11) the trivial bound $M(f) \leq k h(f)$.

Date: 17 August 2016.

2010 Mathematics Subject Classification. 11R06.

Key words and phrases. polynomials, Mahler measure, height, closure. 
Corollary 1. Given $k \geq 1$, there are only finitely many possible choices for integers $a_{1}, \ldots, a_{k}$ such that $M(f)=1$ for some $f(x)=a_{1} z^{n_{1}}+\cdots+a_{k-1} z^{n_{k-1}}+a_{k}$ and any choice of distinct integer exponents $n_{1}, n_{2}, \ldots, n_{k-1}$.

This corollary leaves open the question of whether, for fixed $a_{1}, \ldots, a_{k}$, the number of choices for the exponents $n_{i}$ is finite or infinite. This is discussed in Section 5 .

Theorem 1 in fact holds for Laurent polynomials in several variables, as the next result states. Since it follows quite easily from the one-variable case, we decided to relegate this general case to a corollary. Recall that the logarithmic Mahler measure in the general case is defined for $F=F\left(z_{1}, \ldots, z_{\ell}\right)$ as

$$
m(F)=\int_{0}^{1} \cdots \int_{0}^{1} \log \left|F\left(e^{2 \pi i t_{1}}, \ldots, e^{2 \pi i t_{\ell}}\right)\right| d t_{1} \cdots d t_{\ell}
$$

Again, $M(F):=\exp (m(F))$.

In [1], David Boyd studied the set $\mathcal{L}$ of Mahler measures of polynomials $F$ in any number of variables having integer coefficients. He conjectured that $\mathcal{L}$ is a closed subset of $\mathbb{R}$. Our Theorem 2 below is a result in the direction of this conjecture, but where we restrict the polynomials $F$ under consideration to be the sum of at most $k$ monomials. In [13, Theorem 3], the second author proved another restricted closure result of this kind, where the restriction was, instead, to integer polynomials $F$ of bounded length (sum of the moduli of its coefficients).

Boyd's conjecture is a far-reaching generalisation of a question of D.H. Lehmer [7], who asked whether there exists an absolute constant $C>1$ with the property that, for integer polynomials $f$ in one variable, either $M(f)=1$ or $M(f) \geq C$.

We now state our generalisation of Theorem 1, In it, we write $\mathbf{z}_{\ell}=\left(z_{1}, \ldots, z_{\ell}\right)$.

Corollary 2. Let $F\left(\mathbf{z}_{\ell}\right) \in \mathbb{C}\left[\mathbf{z}_{\ell}\right]$ be a nonzero Laurent polynomial in $\ell \geq 1$ variables that is the sum of $k$ monomials. Then

$$
M(F) \geq \frac{h(F)}{2^{k-2}}
$$

Corollary 2 is an essential ingredient in our next result. For this, we fix $k \geq 1$ and consider the set $\mathcal{H}_{k}$ of Laurent polynomials $F\left(\mathbf{z}_{\ell}\right)=F\left(z_{1}, \ldots, z_{\ell}\right)$ for all $\ell \geq 1$ with integer coefficients that are the sum of at most $k$ monomials. So such an $F$ is of the form

$$
F\left(\mathbf{z}_{\ell}\right)=\sum_{\mathbf{j} \in J} c(\mathbf{j}) \mathbf{z}_{\ell}^{\mathbf{j}}
$$

where $J \subset \mathbb{Z}^{\ell}$ has $k$ column vector elements $\mathbf{j}$, with $\mathbf{z}_{\ell}^{\mathbf{j}}=z_{1}^{j_{1}} \cdots z_{\ell}^{j_{\ell}}$, where $\mathbf{j}=\left(j_{1}, \ldots, j_{\ell}\right)^{\operatorname{tr}}$, and the $c(\mathbf{j})$ 's are integers, some of which could be 0 . The number of variables $\ell$ defining $F$ is unspecified, and can be arbitrarily large. We let $m\left(\mathcal{H}_{k}\right)$ denote the set $\left\{m(F): F \in \mathcal{H}_{k}\right\}$.

Theorem 2. The set $m\left(\mathcal{H}_{k}\right)$ is a closed subset of $\mathbb{R}_{\geq 0}$. Furthermore, 0 is an isolated point of $m\left(\mathcal{H}_{k}\right)$.

In fact the isolation of 0 in $m\left(\mathcal{H}_{k}\right)$ has been essentially known for some time, indeed with explicit lower bounds for the size of the gap between 0 and the rest of the set. The first 
such bound was given for one-variable polynomials by Dobrowolski, Lawton and Schinzel [3]. This was improved by Dobrowolski in [4] and later improved further in [5], where it was shown that for noncyclotomic $f \in \mathbb{Z}[z]$

$$
M(f) \geq 1+\frac{1}{\exp \left(a 3^{\lfloor(k-2) / 4\rfloor} k^{2} \log k\right)},
$$

where $a<0.785$. Arguing as in the proof of Corollary 2 below shows that the gap holds for polynomials in several variables too, and so applies to all $m(F)$ in $m\left(\mathcal{H}_{k}\right) \backslash\{0\}$.

\section{Proof of Theorem 1 and Corollary 1$]$}

We first prove the theorem by induction under the restriction that all $a_{1}, \ldots, a_{k}$ are assumed to be nonzero. We employ two well-known facts:

(i) $M(f)=M\left(f^{*}\right)$ where $f^{*}(z)=z^{n} f\left(z^{-1}\right)$, with $n=\operatorname{deg} f$. This immediately follows from (1).

(ii) $M(f) \geq M\left(\frac{1}{n} f^{\prime}\right)$. This was proved by Mahler in [9] .

For the base case $k=2$ of our induction, we have $M(f)=\max \left\{\left|a_{1}\right|,\left|a_{2}\right|\right\}=h(f)$, as required.

Suppose now that the conclusion of the (restricted) theorem is true for some $k \geq 2$, and suppose that $f$ has $k+1$ nonzero terms, that is, $f(x)=a_{1} z^{n_{1}}+\cdots+a_{k} z^{n_{k}}+a_{k+1}$. Then $f^{*}(z)=a_{k+1} z^{n_{1}}+a_{k} z^{n_{1}-n_{k}}+\cdots+a_{1}$. Because the $a_{i}$ are assumed nonzero, both $f$ and $f^{*}$ have degree $n_{1}$. Suppose that $h(f)=\left|a_{i}\right|$ for some $i,(1 \leq i \leq k+1)$. Then $h\left(\frac{1}{n_{1}} f^{\prime}\right) \geq \frac{n_{i}}{n_{1}} h(f)$, and $h\left(\frac{1}{n_{1}}\left(f^{*}\right)^{\prime}\right) \geq \frac{n_{1}-n_{i}}{n_{1}} h(f)$. Clearly $\max \left\{\frac{n_{i}}{n_{1}}, \frac{n_{1}-n_{i}}{n_{1}}\right\} \geq \frac{1}{2}$, with $f^{\prime}$ and $\left(f^{*}\right)^{\prime}$ having $k$ terms each. Hence, by (i), (ii) and the induction hypothesis

$$
M(f) \geq \max \left\{M\left(\frac{1}{n_{1}} f^{\prime}\right), M\left(\frac{1}{n_{1}}\left(f^{*}\right)^{\prime}\right)\right\} \geq \frac{1}{2} \frac{h(f)}{2^{k-2}}
$$

which completes the inductive step, and the induction argument.

Now we can do the general case. If some of the $a_{i}$ can be 0 , then $f(z)$ is of the form $z^{j} f_{1}(z)$, where $j \geq 0$ and $f_{1}$ is of the form (2), but with $k_{1}$ nonzero terms, where $0<k_{1} \leq k$. Then, using (11),

$$
M(f(z))=M\left(z^{j} f_{1}(z)\right)=M\left(f_{1}(z)\right)
$$

and, since $h\left(f_{1}\right)=h(f)$ we have

$$
M(f)=M\left(f_{1}\right) \geq \frac{h\left(f_{1}\right)}{2^{k_{1}-2}} \geq \frac{h(f)}{2^{k-2}} .
$$

Corollary 1 now follows straight from the theorem, because any such $f$ must have height at most $2^{k-2}$, giving at most $\left(2^{k-1}+1\right)^{k}$ possible choices for $a_{1}, \ldots, a_{k}$.

\section{Proof of Corollary 2}

For the Proof of Corollary 2, we need the following simple result. 
Lemma 1. Let $\mathbf{r}_{n}=\left(1, n, n^{2}, \ldots, n^{\ell-1}\right) \in \mathbb{Z}^{\ell}$. Then for any finite set $V$ of nonzero vectors in $\mathbb{R}^{\ell}$ there is an integer $N$ such that for each $n>N$ the vector $\mathbf{r}_{n}$ is not orthogonal to any vector $\mathbf{v} \in V$.

Proof. Write $\mathbf{v} \in V$ in the form $\mathbf{v}=\left(v_{1}, \ldots, v_{j}, 0, \ldots, 0\right)$ say, where $v_{j} \neq 0$ and $j \leq \ell$. If $j=1$ then $\left|\mathbf{v} \cdot \mathbf{r}_{n}\right|=\left|v_{1}\right|>0$, so assume $j \geq 2$. Then

$$
\begin{aligned}
\left|\mathbf{v} \cdot \mathbf{r}_{n}\right| & =\left|\sum_{i=1}^{j} v_{i} n^{i-1}\right| \\
& \geq\left|v_{j}\right|\left(n^{j-1}-n^{j-2}\left(\sum_{i=1}^{j-1}\left|v_{i} / v_{j}\right|\right)\right) \\
& >0 \text { for } n>\sum_{i=1}^{j-1}\left|v_{i} / v_{j}\right|,=N_{\mathbf{v}} \text { say } .
\end{aligned}
$$

Now take $N=\max _{\mathbf{v} \in V} N_{\mathbf{v}}$.

Following [12], given a fixed integer $s \geq 1$ and a polynomial $F$ in $s$ variables, $\ell \geq 0$ and an $\ell \times s$ matrix $A=\left(a_{i j}\right) \in \mathbb{Z}^{\ell \times s}$, define the $s$-tuple $\mathbf{z}_{\ell}^{A}$ by

$$
\mathbf{z}_{\ell}^{A}=\left(z_{1}, \ldots, z_{\ell}\right)^{A}=\left(z_{1}^{a_{11}} \ldots z_{\ell}^{a_{\ell 1}}, \ldots, z_{1}^{a_{1 s}} \ldots z_{\ell}^{a_{\ell s}}\right)
$$

(which is $(1,1, \ldots, 1) \in \mathbb{Z}^{s}$ when $\ell=0$ ) and $F_{A}\left(\mathbf{z}_{\ell}\right)=F\left(\mathbf{z}_{\ell}^{A}\right)$, a polynomial in $\ell$ variables $z_{1}, \ldots, z_{\ell}$. Then $m\left(F_{A}\right)$ is defined by (3) with $F$ replaced by $F_{A}$. Denote by $\mathcal{P}(F)$ the set $\left\{F_{A}: A \in \mathbb{Z}^{\ell \times s}, \ell \geq 0\right\}$, and by $\mathcal{M}(F)$ the set $\left\{m\left(F_{A}\right): F_{A} \in \mathcal{P}(F), F_{A} \neq 0\right\}$.

In the case $\ell=1$, and with $A$ replaced by $\mathbf{r}=\left(r_{1}, \ldots, r_{s}\right)$, we have $z^{\mathbf{r}}=\left(z^{r_{1}}, \ldots, z^{r_{s}}\right)$ and $F_{\mathbf{r}}(z)=F\left(z^{\mathbf{r}}\right)$.

We also need the following.

Proposition 1. Let $\ell \geq 1, n \geq 1$, and $\mathbf{r}_{n}=\left(1, n, n^{2}, \ldots, n^{\ell-1}\right)$, as in Lemma 1. Then for any Laurent polynomial $F\left(\mathbf{z}_{\ell}\right)$ in $\ell$ variables $\mathbf{z}_{\ell}=\left(z_{1}, \ldots, z_{\ell}\right)$ we have $m\left(F_{\mathbf{r}_{n}}(z)\right) \rightarrow$ $m\left(F\left(\mathbf{z}_{\ell}\right)\right)$ as $n \rightarrow \infty$. Furthermore, for $n$ sufficiently large, $h\left(F_{\mathbf{r}_{n}}\right)=h(F)$.

Proof. The first part follows from results of Boyd [2, p. 118] and Lawton [6]; see also [13, Lemma 13 and Proposition 14]. Next, note that $F$ is the sum of $k$ monomials of the form $c(\mathbf{j}) \mathbf{z}_{\ell}^{\mathbf{j}}$, so that $F_{\mathbf{r}}$ is the sum of $k$ monomials of the form $c(\mathbf{j})\left(z^{\mathbf{r}}\right)^{\mathbf{j}}=c(\mathbf{j}) z^{\mathbf{r} \mathbf{j}}=a_{i} z^{t_{i}}$ say, for some $i$, where $\mathbf{j} \in J$ is a column vector. We now take $\mathbf{r}=\mathbf{r}_{n}$, and apply Lemma 1 to the set $V$ of all nonzero differences $\mathbf{j}-\mathbf{j}^{\prime}$ between elements of $J$. The lemma then guarantees that, for $n$ sufficiently large, the $t_{i}$ are distinct, so that $F_{\mathbf{r}}$ and $F$ have the same coefficients. In particular, $h\left(F_{\mathbf{r}}\right)=h(F)$.

Proof of Corollary [2. This now follows from Theorem 1, using the fact, from Proposition 1, that, for any $\varepsilon>0, F$ has the same height and the same number of monomials as some one-variable polynomial $F_{\mathbf{r}}$ with $\left|m\left(F_{\mathbf{r}}\right)-m(F)\right|<\varepsilon$. 


\section{Proof of Theorem 2}

Proof of Theorem Q Throughout, $k \geq 2$ is fixed, while $\ell \geq 0$ can vary. Take any $F \in \mathcal{H}_{k}$, with $F\left(\mathbf{z}_{\ell}\right)=\sum_{\mathbf{j} \in J} c(\mathbf{j}) \mathbf{z}_{\ell}^{\mathbf{j}}$, say, where $J$ is a $k$-element subset of $\mathbb{Z}^{\ell}$. Then $F \in \mathcal{P}\left(a_{1} z_{1}+\right.$ $\left.\cdots+a_{k} z_{k}\right)$ for some integers $a_{i}$, where $\{c(\mathbf{j})\}_{\mathbf{j} \in J}=\left\{a_{i}\right\}_{i=1, \ldots, k}$ as multisets. (Again, some $a_{i}$ 's could be 0 .) Conversely, every element of $\mathcal{P}\left(a_{1} z_{1}+\cdots+a_{k} z_{k}\right)$ is a sum of $k$ monomials. (Note that because monomial terms may combine to form a single monomial term, or indeed vanish, the resulting $a_{i}$ 's for some polynomials in $\mathcal{P}\left(a_{1} z_{1}+\cdots+a_{k} z_{k}\right)$ may be different from the $a_{i}$ 's that we started with. Because of this, the height of some such polynomials may be larger or smaller than the height $\max _{i=1}^{k}\left|a_{i}\right|$ of $a_{1} z_{1}+\cdots+a_{k} z_{k}$. This does not matter, however!)

Next, take some bound $B>0$ and consider all $F$ such that $m(F) \leq B$. Then, by Corollary 2,

$$
h(F) \leq 2^{k-2} e^{B},
$$

so that there are only finitely many choices for the integers $a_{i}$. So $m(F)$ belongs to the union - call it $U_{B}$ - of finitely many sets $\mathcal{M}\left(a_{1} z_{1}+\cdots+a_{k} z_{k}\right):=m\left(\mathcal{P}\left(a_{1} z_{1}+\cdots+a_{k} z_{k}\right)\right)$, intersected with the interval $[0, B]$. Thus $U_{B}$ is closed since, by [13, Theorem 1], each set $\mathcal{M}\left(a_{1} z_{1}+\cdots+a_{k} z_{k}\right)$ is closed. Note that the finite number of sets comprising $U_{B}$ depends on $k$ and on $B$, but not on $F$. Finally, we see that $m\left(\mathcal{H}_{k}\right)$ is closed. This is because any convergent sequence in $m\left(\mathcal{H}_{k}\right)$, being bounded, belongs, with its limit point, to $U_{B}$ for some $B$.

Finally, to show that 0 is an isolated point of $m\left(\mathcal{H}_{k}\right)$, note that, by [13, Theorem 2] it is an isolated point of every $\mathcal{M}\left(a_{1} z_{1}+\cdots+a_{k} z_{k}\right)$ that contains 0 . Hence, since $0 \in U_{B}$ for every $B>0$, it is an isolated point of $U_{B}$ and therefore also of $m\left(\mathcal{H}_{k}\right)$.

\section{Products of Cyclotomic polynomials that have the same coefficients}

In this section we address the question of whether two or more integer polynomials having Mahler measure 1 (and so being products of cyclotomic polynomials $\Phi_{n}(z)$ ) can have the same set of $k$ nonzero coefficients. We restrict our attention to the case where all the coefficients $a_{i}$ are 1 . This already indicates what can happen.

Let $k \geq 2$ and define

$$
S=\left\{\left(n_{1}, \ldots, n_{k-1}\right) \in \mathbb{Z}^{k-1} \mid n_{1}>n_{2}>\cdots>n_{k-1}>0 \text { with } \operatorname{gcd}\left(n_{1}, \ldots, n_{k-1}\right)=1\right\} .
$$

Proposition 2. For $\mathbf{n} \in S$ define $f_{\mathbf{n}}(x)=z^{n_{1}}+\cdots+z^{n_{k-1}}+1$. Let

$$
S_{c}=\left\{\mathbf{n} \in S \mid M\left(f_{\mathbf{n}}\right)=1\right\} .
$$

The set $S_{c}$ is finite if and only if $k$ is a prime number.

Since for instance $\Phi_{5}(z)$ and $\Phi_{5}(z) \Phi_{6}(z)$ have the same nonzero coefficients, $S_{\mathrm{c}}$ can, however, contain more than one element for $k$ prime.

Proof. Consider first the case of composite $k$.

Suppose that $k=s t$, where integers $s$ and $t$ are greater than 1 . Let $g(z)=\sum_{j=0}^{s-1} z^{j}$ and 
$h(x)=\sum_{j=0}^{t-1} z^{j}$. If $m$ and $l$ are arbitrary integers greater than 1 and such that $\operatorname{gcd}(m, l)=$ $\operatorname{gcd}(m, t !)=\operatorname{gcd}(l, s !)=1$ then it is not difficult to check that $g\left(z^{m}\right) h\left(z^{l}\right)=f_{\mathbf{n}}(z)$ for some $\mathbf{n} \in S$. Since $M\left(g\left(z^{m}\right)\right)=M\left(h\left(z^{l}\right)\right)=1$, we see that in fact $f_{\mathbf{n}}(z) \in S_{\mathrm{c}}$, and so $S_{\mathrm{c}}$ is infinite.

Now suppose that $k=p$ is prime.

Let $\mathbf{n} \in S_{\mathrm{c}}$ and consider $f_{\mathbf{n}}(z)=z^{n_{1}}+\cdots+z^{n_{k-1}}+1$. By Kronecker's Theorem, $f_{\mathbf{n}}$ is a product of cyclotomic polynomials. Further, $f(1)=p$. However the value of a cyclotomic polynomial at 1 is $\Phi_{m}(1)=1$ if $m$ is divisible by two distinct primes or $\Phi_{m}(1)=q$ if $m$ is a power of a single prime $q$. Thus, for some $n, \Phi_{p^{n}}$ divides $f$.

We claim that $n=1$. To show this, we use a theorem of Mann [11] which, in our notation, takes the form of the following lemma.

Lemma 2. Let $f(z)=\sum_{i=1}^{k-1} a_{i} z^{n_{i}}+a_{k} \in \mathbb{Z}[z]$, with $\left(n_{1}, \ldots, n_{k-1}\right) \in S$ and where the coefficients $a_{i}, 1 \leq i \leq k$ nonzero. If a cyclotomic polynomial $\Phi$ divides $f$ but $\Phi$ does not divide any proper subsum of $\sum_{i=1}^{k-1} a_{i} z^{n_{i}}+a_{k}$ then $\Phi=\Phi_{q}$, where $q$ is squarefree and composed entirely of primes less than or equal to $k$.

In the case of $f_{\mathbf{n}}$, a proper subsum defines a polynomial $g$ such that $g(1)$ counts its number of monomials. Hence $g(1)<p$, and consequently $g$ cannot be divisible by $\Phi_{p^{n}}$. By Lemma 2, $p^{n}$ is squarefree, so $n=1$, as claimed.

Next, we need the following result.

Theorem 3 (Dobrowolski [5, Theorem 2 and Corollary 1]). Let $f(z)=\sum_{i=1}^{k} a_{i} z^{n_{i}} \in \mathbb{Z}[z]$, $f(0) \neq 0$, be a polynomial with $k$ nonzero coefficients. There are positive constants $c_{1}$ and $c_{2}$, depending only on $k$, and polynomials $f_{0}, f_{2} \in \mathbb{Z}[z]$ such that if

$$
\operatorname{deg} f_{c} \geq\left(1-\frac{1}{c_{1}}\right) \operatorname{deg} f
$$

then either

$$
f(z)=f_{0}\left(z^{l}\right), \quad \text { where } \operatorname{deg} f_{0} \leq c_{2},
$$

or

$$
f(z)=\left(\prod_{i} \Phi_{q_{i}}\left(z^{l_{i}}\right)\right) f_{2}(z), \text { where } \min _{i}\left\{l_{i}\right\} \geq \max \left\{\frac{1}{2 c_{1}} \operatorname{deg} f, \operatorname{deg} f_{2}\right\} .
$$

Furthermore in this second case then $f_{2}(z)= \pm \sum_{i=j}^{k} a_{i} z^{n_{i}}$ for some $j$ with $1<j \leq k$.

In this theorem $\Phi_{q}$ is the $q$-th cyclotomic polynomial, while $f_{\mathrm{c}}$ is the product of all cyclotomic polynomials dividing $f$.

Now we apply Theorem [3. If equation (44) of its conclusion occurs then, with our restriction on $S, \operatorname{deg} f \leq c_{2}$. If equation (5) occurs, then either $\operatorname{deg} f \leq 2 c_{1}$ or $\min \left\{l_{i}\right\} \geq 2$. In the latter case $\Phi_{p}$ must divide $f_{2}$, where $f_{2}$ is a proper subsum of $f$. Hence $f_{2}(1)<p$, contradicting $\Phi_{p} \mid f_{2}$. Thus in all admissible cases the degree of $f$ is bounded by a constant depending only on $k$. Therefore $S_{\mathrm{c}}$ is finite. 


\section{REFERENCES}

[1] D.W. Boyd, Speculations concerning the range of Mahler's measure, Canad. Math. Bull. 24 (1981), no. 4, 453-469.

[2] _ Kronecker's theorem and Lehmer's problem for polynomials in several variables, J. Number Theory 13 (1981), no. 1, 116-121.

[3] E. Dobrowolski, W. Lawton and A. Schinzel, On a problem of Lehmer, in Studies in pure mathematics (Birkhäuser, Basel, 1983), pp. 135-144.

[4] E. Dobrowolski, Mahler's measure of a polynomial in function of the number of its coefficients, Canad. Math. Bull. 34 (1991), no. 2, 186-195.

[5] _ Mahler's measure of a polynomial in terms of the number of its monomials, Acta Arith. 123 (2006), no. 3, 201-231.

[6] W. Lawton, A problem of Boyd concerning geometric means of polynomials, J. Number Theory 16 (1983), no. 3, 356-362.

[7] D.H. Lehmer, Factorization of certain cyclotomic functions, Ann. of Math. (2) 34 (1933), no. 3, 461-479.

[8] K. Mahler, An application of Jensen's formula to polynomials, Mathematika 7 (1960), 98-100.

[9] - On the zeros of the derivative of a polynomial, Proc. Roy. Soc. London Ser. A 264 (1961), $145-154$.

[10] 341-344.

[11] H.B. Mann, On linear relation between roots of unity, Mathematika 12 (1965), 107-117.

[12] A. Schinzel, Polynomials with special regard to reducibility, with an appendix by Umberto Zannier, Encyclopedia of Mathematics and its Applications, Vol. 77 (Cambridge University Press, Cambridge, 2000).

[13] C.J. Smyth, Closed sets of Mahler measures, arXiv:1606.04338 [math.NT].

Department of Mathematics and Statistics, University of Northern British Columbia, Prince George, BC, Canada

E-mail address: edward.dobrowolski@unbc.ca

School of Mathematics and Maxwell Institute for Mathematical Sciences, University of Edinburgh, Edinburgh EH9 3FD, Scotland, U.K.

E-mail address: c.smyth@ed.ac.uk 